Meta

Journal des tradlucteurs

Translators' Journal

\title{
La Banque de terminologie du gouvernement canadien et l'ATIO
}

\section{Nelson Rodrigue}

Volume 28, numéro 2, juin 1983

URI : https://id.erudit.org/iderudit/003970ar

DOI : https://doi.org/10.7202/003970ar

Aller au sommaire du numéro

Éditeur(s)

Les Presses de l'Université de Montréal

ISSN

0026-0452 (imprimé)

1492-1421 (numérique)

Découvrir la revue

Citer cette note

Rodrigue, N. (1983). La Banque de terminologie du gouvernement canadien et l'ATIO. Meta, 28(2), 215-217. https://doi.org/10.7202/003970ar d'utilisation que vous pouvez consulter en ligne.

https://apropos.erudit.org/fr/usagers/politique-dutilisation/ 


\section{LA BANQUE DE TERMINOLOGIE DU GOUVERNEMENT CANADIEN ET L'ATIO*}

La Banque de terminologie est constituée des quatre fichiers suivants: le fichier Terminologie, le fichier Titres et appellations officielles, le Dictionnaire automatique et le fichier Recherche.

La structure du premier de ces fichiers, le fichier Terminologie, repose sur trois principes: l'uninotionnalité, la valeur des équivalences proposées et l'uniformité des méthodes.

Le fichier Terminologie est uninotionnel, c'est-à-dire qu'une fiche ne contient qu'une seule notion et qu'une notion ne fait l'objet que d'une seule fiche. Il comporte trois volets (A, B et $\mathrm{C}$ ), dont chacun correspond à un indice de fiabilité, afin de permettre d'emmagasiner efficacement le fruit des recherches et d'avertir l'usager de la fiabilité des renseignements qui lui sont fournis.

Le fichier Titres et appellations officielles renferme les fiches dont les vedettes sont constituées de noms propres ou de désignations assimilables à des noms propres: appellations commerciales, personnes morales, unités administratives, titres, distinctions honorifiques, noms géographiques, noms de peuples et de nationalités, etc.

Le Dictionnaire automatique, contrairement aux autres fichiers de la Banque de terminologie, ne contient pas de justifications, c'est-àdire pas de définitions ni de contextes. Il sera alimenté principalement par voie de chargements massifs de lexiques bilingues mis au point par des organismes officiels. Des fiches individuelles produites par des terminologues peuvent également y être versées.

\footnotetext{
* Colloque de Glendon, 1980.
}

Le fichier Recherche est formé de l'actuel fichier provisoire Termium I, du fruit de dépouillements de traductions, de demi-fiches, de fiches incomplètes faites par les terminologues de la Direction générale de la terminologie et de la documentation qui ne sont pas rigoureusement conformes à nos méthodes de travail. En pratique, ce fichier est alimenté surtout par des chargements massifs (lots de fiches, documents avec justifications). Selon leur nature, toutes les fiches de ce fichier sont destinées à en sortir pour passer soit dans les volets $\mathrm{A}, \mathrm{B}$ ou $\mathrm{C}$ du fichier Terminologie, soit dans le fichier Titres et appellations officielles ou dans le Dictionnaire automatique. Contrairement au fichier Dictionnaire automatique, le fichier Recherche peut contenir des fiches avec justifications.

Au 31 mars 1980, la Banque de terminologie contenait quelque 720000 fiches réparties entre les divers fichiers.

À l'heure actuelle, le taux de réponse de la Banque de terminologie se situe, en moyenne, aux environs de $\mathbf{4 5}$ pour cent. L'objectif que nous nous sommes fixé pour les prochaines années est de faire passer ce taux à 70 pour cent. Nous espérons y arriver surtout en emmagasinant massivement dans l'ordinateur les lexiques, dictionnaires, vocabulaires, fiches, etc. que les traducteurs, les terminologues et nos collaborateurs nous auront recommandés. Par ailleurs, les échanges de données avec d'autres banques ou des organismes producteurs de terminologie devraient contribuer considérablement à nous rapprocher de l'objectif. Nous estimons toutefois qu'il sera difficile de dépasser un taux de 80 ou 90 pour cent à cause d'un ensemble de facteurs: inaccessibilité de certaines publications, usage oral, décalage entre langues, etc.

Le réseau est aujourd'hui formé de 80 terminaux. Ils sont situés à Ottawa, à Montréal, à 
Québec, à Saint-Jean (Québec), à Moncton, à Toronto, à Frédéricton et à Winnipeg. Nous avons fait l'acquisition en 1977 de deux terminaux lourds (l'un installé à Ottawa, l'autre à Montréal) qui permettent la production de listages servant de vocabulaires et d'autres sorties volumineuses. Ces terminaux lourds ont une capacité d'impression de 900 lignes/minutes, à comparer à 30 caractères/seconde pour les terminaux légers du réseau.

Contrairement à ce que beaucoup de gens pensent, la Banque de terminologie du gouvernement canadien n'est pas un service public, du moins pas encore. Elle a pour mandat, tout comme le Bureau des traductions, l'organisme qui l'administre, d'aider à répondre aux besoins de traduction, d'interprétation et de terminologie du Parlement canadien et des ministères et organismes fédéraux qui en dépendent. Cependant, comme c'est le cas pour les banques commerciales, la viabilité de la Banque de terminologie du gouvernement canadien est directement fonction des dépôts qui y sont faits ou, si l'on préfère, de son alimentation. Si l'alimentation n'est assurée que par le personnel du Bureau des traductions, on n'aura jamais qu'un outil d'une extrême puissance mais sans grande utilité, car il faudrait disposer d'une armée de terminologues pour tenir à jour les données, ce qui est utopique. La participation de l'ensemble des praticiens est essentielle. Il serait, en effet, inutilement coûteux de refaire ce que d'autres ont déjà fait.

Par conséquent, bien qu'elle ne soit pas encore habilitée à étendre ses services à l'ensemble de la profession, voire de la population canadienne, la Banque de terminologie n'en est pas moins désireuse de conclure des échanges de données.

C'est dans cette perspective que, même s'il n'est pas un service public, le Bureau des traductions a pu justifier l'abonnement de gouvernements provinciaux, d'organismes publics ou semi-publics, ou même d'entreprises privées, à la Banque de terminologie. Parmi les abonnés à l'extérieur du Bureau des traductions, citons le Bureau de traduction du gouvernement du Nouveau-Brunswick, Téléglobe Canada, le Canadien National, Air Canada, Canadien Pacifique, l'Organisation de l'aviation civile internationale (OACI), l'Université de Moncton. Tous ces abonnés de la Banque à l'extérieur comme à l'intérieur du Bureau des traductions et de la Fonction publique canadienne constituent ce que nous appelons le réseau de terminologie du gouvernement canadien. Quand le réseau aura pris une certaine expansion, on pourra s'adresser à tel ou tel point spécialisé pour toute recherche de pointe; en retour, chaque point spécialisé pourra faire appel aux autres points du réseau pour obtenir de l'aide dans les domaines qui ne sont pas leurs spécialités.

D'autre part, la Banque de terminologie envisage des échanges de données avec des organismes internationaux tels que l'ONU, l'UNESCO et la Banque mondiale. Nous avons amorcé dernièrement un échange avec EURODICAUTOM, la banque de terminologie de la Commission des communautés économiques à Luxembourg. Nous avons obtenu il y a quelques mois la terminologie spécialisée d'Intelsat. À l'occasion d'une mission documentaire réalisée en France en 1978, nous avons obtenu de grandes quantités de fiches dans divers domaines de recherche prioritaires et notamment celles de l'OCDE dans le domaine nucléaire. Nous participons aux séances de normalisation du vocabulaire de l'OTAN. Depuis environ trois ans, nous avons des représentants au sein de divers comités de l'Organisation internationale de normalisation (ISO), y compris le ISO/TC 37 (coordination et principes de terminologie). Enfin, nous fondons beaucoup d'espoir sur les travaux d'INFOTERM, organisme dont le siège est à Vienne et qui vise à organiser les échanges entre banques de terminologie.

C'est aussi dans la perspective des échanges de données que nous envisageons notre collaboration avec les membres de l'ATIO et les traducteurs de Toronto. Nous sommes convaincus que les membres de l'ATIO et particulièrement ceux qui œuvrent ici dans la région de Toronto et qui sont spécialisés dans un certain nombre de domaines précis (assurances, électronique, finances, industric minière, etc.), pourraient contribuer considérablement à l'enrichissement de la Banque dans leurs langues de spécialités.

Voyons maintenant comment, concrètement, la collaboration avec les membres de l'ATIO pourrait se réaliser.

Il est un moyen d'accès à la Banque de terminologie qui n'est pas encore au point mais qui, je crois, pourrait sans doute être mis en place assez rapidement si les traducteurs de Toronto y trouvaient un certain intérêt. Si nous ne nous y sommes pas encore vraiment intéressés, c'est sans doute parce que nous n'avons pas eu besoin d'y recourir jusqu'ici. Je veux parler ici de la microfiche, moyen très peu coûteux d'avoir accès à toutes sortes d'informations. Le Bureau des traductions n'a pas jusqu'à maintenant senti le besoin d'exploiter la microfiche pour la simple raison que plus de $95 \%$ de ses traducteurs ont déjà accès directement à la Banque de terminologie par terminal. Cependant, selon le principe que la fonction crée l'organe, si l'ATIO, au nom d'un certain nombre de ses membres, disons au moins une dizaine, était prête à adresser au Bureau des traductions une demande ferme afin que nous envisagions de donner aux intéressés accès, par ce moyen, au contenu de la Banque, nous pourrions peut-être faire le néces- 
saire pour mettre au point un système de microfiches.

Le Bureau des traductions pourrait aussi songer à offrir aux traducteurs intéressés un service de sorties d'ordinateur en différé. Autrement dit, nous pourrions fournir, sur demande, des lexiques portant sur différents domaines d'intérêt. Le coût d'impression approximatif de chaque terme et des quelques données afférentes étant de $1 c$, un lexique de 5000 termes reviendrait donc à environ $\$ 50$.

Autre moyen d'accès à la Banque de terminologie pour les membres de l'ATIO : l'abonnement, c'est-à-dire l'acquisition d'un terminal permettant la consultation directe des fichiers. Les abonnements sont régis par la politique d'expansion de la Banque de terminologie qui a été établie il y a environ dix-huit mois par un groupe d'étude que j'ai eu l'honneur de présider. La politique en question réitère le principe de la «coopérative» voulant que ceux qui sont intéressés à adhérer au réseau de terminologie s'engagent, entre autres, à contribuer à l'enrichissement et à l'épuration de la Banque.

Chaque demande d'accès direct à la Banque de terminologie est examinée par le Conseil de la Direction générale de la terminologie et de la documentation. Le demandeur doit, dans ses démarches, faire la preuve de l'utilité de la Banque pour son service ou son entreprise et préciser qu'il est prêt à se conformer aux modalités habituelles de collaboration: cession de son fonds terminologique à la Banque pour emmagasinement; contribution à l'alimentation et à l'épuration de la Banque de terminologie, principalement dans ses domaines d'intérêt; utilisation dans la mesure du possible des instruments et méthodes de travail en terminologie du Bureau des traductions. La présence d'un ou plusieurs terminologues sur place est un autre élément pris en considération dans l'étude des demandes d'accès à la Banque. Tous les abonnements sont assujettis à une période d'essai de six mois au terme de laquelle il est procédé à une évaluation conjointe de la collaboration.

Il n'est pas question d'abonnement payant à la Banque de terminologie, le seul paiement accepté prenant la forme d'un apport terminologique. L'abonné n'assume pour ainsi dire que les frais d' «expédition» de la terminologie, c'est-à-dire les frais d'achat ou de location d'un terminal et d'un modem, les frais téléphoniques et les frais d'ordinateur. Ceux qui s'intéressent à un abonnement peuvent écrire à l'adresse suivante: directeur général de la Terminologie et de la Documentation, Bureau des traductions, Ottawa, K1A 0M5.

Comme vous le voyez, il y a trois moyens pour vous d'avoir accès au contenu de la Banque de terminologie : premièrement, l'abonnement en bonne et due forme, c'est-à-dire le raccordement par terminal; deuxièmement, la microfiche, mode d'accès à mettre au point; et, troisièmement, les imprimés d'ordinateur portant sur des domaines particuliers.

Dans l'optique d'une mise en commun des ressources terminologiques canadiennes et de l'élimination du double emploi si coûteux en terminologie, nous jugeons essentiel qu'une éventuelle collaboration avec vous prenne la forme de véritables échanges de données, et ne soit pas une collaboration à sens unique.

NeLSON Rodrigue 